\title{
An Efficient Method for Extraction and Enrichment of $\gamma$ - Linolenic Acid (GLA) from Spirulina
}

\author{
Ali Choopani ${ }^{1,2, *} \mathbb{( D )}$, Mohammad Fazilati ${ }^{1}{ }^{(\mathbb{D}}$, Ali Mohammad Latifi ${ }^{2}{ }^{(\mathbb{D})}$, Hossain Salavati ${ }^{1(\mathbb{D})}$, \\ Mohammad Reza Khodabakhshi ${ }^{2}$ (D), Mahmood Fadaie ${ }^{3 \text { (D) }}$
Department of Biochemistry, Faculty of Biologic Science, Payame Noor University, Tehran, Iran Applied Biotechnology Research Center, Baqiyatallah University of Medical Sciences, Tehran, Iran
Department of Medical Biochemistry, Faculty of Allied Medicine, Iran University of Medical Sciences Tehran, Iran \\ * Correspondence: choopani.ali3266@gmail.com (A.C.);
}

Received: 9.03.2021; Revised: 28.05.2021; Accepted: 3.06.2021; Published: 27.06.2021

\begin{abstract}
GLA) is useful for improving and treating various diseases, including immune system-related diseases. In the world of commerce, GLA production has problems that compel scientists to search for a simpler, safer, and less costly way. Some microalgae have been introduced as a rich source of fatty acids; Spirulina platensis has attracted scientists due to the presence of $31 \%$ GLA in its fatty acid profile. In the present study, the Iranian strain of spirulina was investigated. Cell culture was performed under optimized conditions and after dry biomass production; the powder was used to continue the study. Finally, the extraction and enrichment steps were performed, and the IR spectra and gas chromatography methods were used for identification and final measurements, respectively. After enrichment by one-step urea crystallization, the percentage of GLA enriched in the total lipid was increased to $61.17 \%$. These results suggest that employing simple, relatively inexpensive, and modified methods for the GLA isolation from the Iranian strain of spirulina may have a potential for industrialization.
\end{abstract}

Keywords: gamma-linolenic acid; Spirulina platensis; fatty acid extraction; fatty acid methyl ester; urea crystallization.

(C) 2021 by the authors. This article is an open-access article distributed under the terms and conditions of the Creative Commons Attribution (CC BY) license (https://creativecommons.org/licenses/by/4.0/).

\section{Introduction}

$\gamma$-linolenic acid (GLA) or "cis 6, 9, 12-octadecatrienoic acid" is an essential fatty acid from the omega- 6 series that should be present in our diet to maintain health. GLA deficiency has been associated with different physiologic/pathophysiologic conditions, including aging, diabetes, alcoholism, atopic dermatitis, premenstrual syndrome, rheumatoid arthritis, cancer, and cardiovascular disease. Due to its different applications in the food, nutraceutical, and pharmaceutical industries [1]. GLA has received more attention in recent years [2]. GLA is found in human milk and small amounts in other common foods such as organ meats; therefore, with the increasing need for this fatty acid, new sources such as evening primrose seeds, blackcurrant, borage, fungi, and spirulina have been introduced [3, 4]. Oil from the seeds of evening primrose is currently the major source of GLA, which is relatively expensive and has possible side effects such as headache, nausea, diarrhea, and rare complications include allergies and seizures; therefore, the commercialization of safe resources is very important $[5$, 6]. Microalgae, particularly spirulina, have been introduced as a rich source for GLA [7]. The 
percentage of fatty acid composition in different spirulina strains is presented in Table 1. Spirulina platensis is the most dominant species of spirulina that is cultivated commercially throughout the world. This species has advantages over other GLA sources, which makes it preferable for commercialization. Spirulina platensis accumulate a significant amount of GLA (31\% of total fatty acids (TFAs) and $1.0-1.4 \%$ dry cell wt.) [8]. They have rapid reproduction and require much fewer land areas compared to conventional crops [9]. There is no enzyme with $\Delta 15$ desaturase activity in Spirulina platensis, therefore alpha isomer of linolenic acid is not synthesized; this makes it easier to purify GLA. Spirulina platensis production is relatively inexpensive and simple since its growth requires only minerals and light energy. It is resistant to unfavorable environmental conditions and also does not cause acute and chronic toxicities, making it safe for human consumption $[10,11]$.

Table 1. Fatty acid composition of 35 spirulina strains grown under standard conditions [12].

\begin{tabular}{l|l} 
Fatty acid & Value \\
\hline $\mathrm{C} 16: 0$ & $42.3-47.6 \%$ \\
\hline $\mathrm{C} 16: 1$ & $2.4-5.4 \%$ \\
\hline $\mathrm{C} 18: 0$ & $0.0-2.1 \%$ \\
\hline $\mathrm{C} 18: 1$ & $2.9-11.8 \%$ \\
\hline $\mathrm{C} 18: 2$ & $13.1-31.5 \%$ \\
\hline$\gamma-\mathrm{C} 18: 3$ & $12.9-29.4 \%$
\end{tabular}

It is well known that GLA content of Spirulina platensis can be increased by altering parameters such as light intensity, carbon source [13], aeration rate [14], and temperature [15]. Also, its cultivation under stress conditions, including salt stress and titanium dioxide $\left(\mathrm{TiO}_{2}\right)$ induced stress [16], can effectively increase the content of GLA. Therefore, by optimizing these parameters and conditions, GLA production will increase further. Comparative studies reported that sonication and microwave ovens are the most effective methods in cell disruption for lipid extraction from microalgae $[17,18]$. In recent years, various methods have been employed to extract and purify GLA; the Folch method, Bligh and Dyer method, and Superior solvent extraction methods are the methods used to extract lipids, particularly GLA, from spirulina [19, 20]. Also, for purification of GLA, methods such as argentated silica gel column chromatography consist of four main steps [21, 22], low-temperature crystallization method [23], and lipase-catalyzed enzymatic purification [24] have been performed. Since high concentrations of GLA are required for dietary and pharmaceutical applications, the extraction and purification process should be considered simple and cost-effective. In this paper, with the optimization of cultivation conditions and employing modified extraction and purification processes, the recovery of highly purified GLA from the Iranian strain of Spirulina platensis has been reported.

\section{Materials and Methods}

\subsection{Sampling.}

Spirulina platensis was isolated in the northern region of Iran; called Alma Ghol Lake, with geographical coordinates of $37^{\circ} 21^{\prime} 00^{\prime \prime} \mathrm{N} 54^{\circ} 34^{\prime} 59^{\prime \prime} \mathrm{E}$. Then, the sample was sent to the Research Institute of Medicinal Plants, Shahid Beheshti University, for identification, and it was reported that this species is Spirulina Platensis. All the materials required for cultivation and measurement were obtained from Merck Chemical Co. (Germany). Experiments were performed under optimized conditions for the production of biomass. 


\subsection{Culture.}

The microalgal biomass was harvested and thoroughly washed with distilled water. The thoroughly washed harvest was dried and stored as a powder in an impervious container at -20 ${ }^{\circ} \mathrm{C}$. Lipid extraction was according to the modified Bligh-Dyer method reported by Takagi et al. $[25,26]$.

\subsection{Extracting.}

4gr of freeze-dried biomass was vigorously mixed with methanol/chloroform/water solution (200:100:80 ml) and sonicated for $20 \mathrm{~min}$. The mixture was vacuum-filtered by suction filtration through Whatman No. 3 filter paper. The supernatant was centrifuged at 4000 rpm for $10 \mathrm{~min}$, treated with $50 \mathrm{ml}$ chloroform and $50 \mathrm{ml}$ water, then the layer containing the lipid was separated using a separating funnel (residual moisture in the chloroform layer was removed by addition of anhydrous sodium sulfate) [27, 28]. Finally, chloroform was removed under vacuum in a rotary evaporator, and the remainder was weighed as lipid.

\subsection{Preparation.}

The lipids were transited into fatty acid methyl esters (FAME) by refluxing the algal lipid with a $2 \%$ aqueous solution of $\mathrm{H}_{2} \mathrm{SO}_{4}$ in ethanol at $80 \mathrm{C}$ for $5 \mathrm{~h}$. The refluxed sample was cooled to room temperature, and the FAME was extracted in ethyl acetate fraction using a separating funnel. Anhydrous sodium sulfate was used to trap the residual moisture present in FAME and was then concentrated under vacuum distillation.

\subsection{Analysis.}

IR spectra were recorded on a Tensor 27 FTIR Spectrometer [29-31] using $\mathrm{CH} 2 \mathrm{Cl} 2$. The spectra of liquid samples were recorded by dissolving in $\mathrm{CH}_{2} \mathrm{Cl}_{2}$. The fatty acid methyl ester analysis was performed on a Varian gas chromatograph equipped with an FID and the capillary column BPX-70 in techno-azma laboratory (Tehran, Iran).

\subsection{Enrichment.}

Urea and ethanol were mixed and refluxed to this boiling mixture; FAME was added and refluxed at $65^{\circ} \mathrm{C}$ with constant stirring to obtain a clear solution which was subsequently cooled to room temperature and was transferred to an ice bath $\left(0{ }^{\circ} \mathrm{C}\right)$. This mixture was incubated at $0{ }^{\circ} \mathrm{C}$ overnight to obtain urea inclusion complexes. The overnight stored mixture was centrifuged at $0{ }^{\circ} \mathrm{C}$ and $7500 \mathrm{rpm}$. The upper liquid phase was carefully collected, and the methyl esters from the liquid phase were extracted in hexane. Anhydrous sodium sulfate was used to dry the residual moisture left in the extracted methyl ester and concentrated under a vacuum. The final FAME concentrate obtained was weighed, and the recovery of GLA was calculated comparing with the original amount of GLA present in microalgal lipid.

\section{Results and Discussion}

\subsection{Result of cultivation optimization.}

The optimal production of GLA by Spirulina platensis requires optimization of their cultivation, and this is very important for their commercialization. The optimized conditions 
for obtaining the largest biomass have been investigated in previous studies and are presented in Table 2.

Table 2. Optimized parameters for the cultivation of Spirulina platensis.

\begin{tabular}{l|l} 
Parameter & Value \\
\hline $\mathrm{PH}$ & 10.5 \\
\hline Temperature & $25{ }^{\circ} \mathrm{C}$ \\
\hline Light intensity & $3500 \mathrm{Lux}$ \\
\hline Culture medium & $\mathrm{NaHCO}_{3}(7.8 \mathrm{gr}), \mathrm{K}_{2} \mathrm{HPO}_{4}(0.5 \mathrm{gr}), \mathrm{NaNO}_{3}(1.8 \mathrm{gr})$, \\
& $\mathrm{K}_{2} \mathrm{SO}_{4}(0.5 \mathrm{gr}), \mathrm{NaCl}(0.5 \mathrm{gr}), \mathrm{MgSO}_{4}(0.4 \mathrm{gr}), \mathrm{CaCl}_{2}(0.4 \mathrm{gr})$, \\
& $\mathrm{FeSO}_{4}(0.4 \mathrm{gr})$ and $\mathrm{EDTA}(0.08 \mathrm{gr})$
\end{tabular}

\subsection{Characterization analysis.}

The biomass obtained was freeze-dried, finely powdered, and lipid was extracted by the modified Bligh and Dyer method. In this method, sonication was used to break the cell wall. The lipid extracted directly was converted to fatty acid esters, and then the presence and percentage of GLA were monitored by IR spectra and gas chromatography. Table 3 shows that the amount of GLA present in the total lipids is $17.37 \%$.
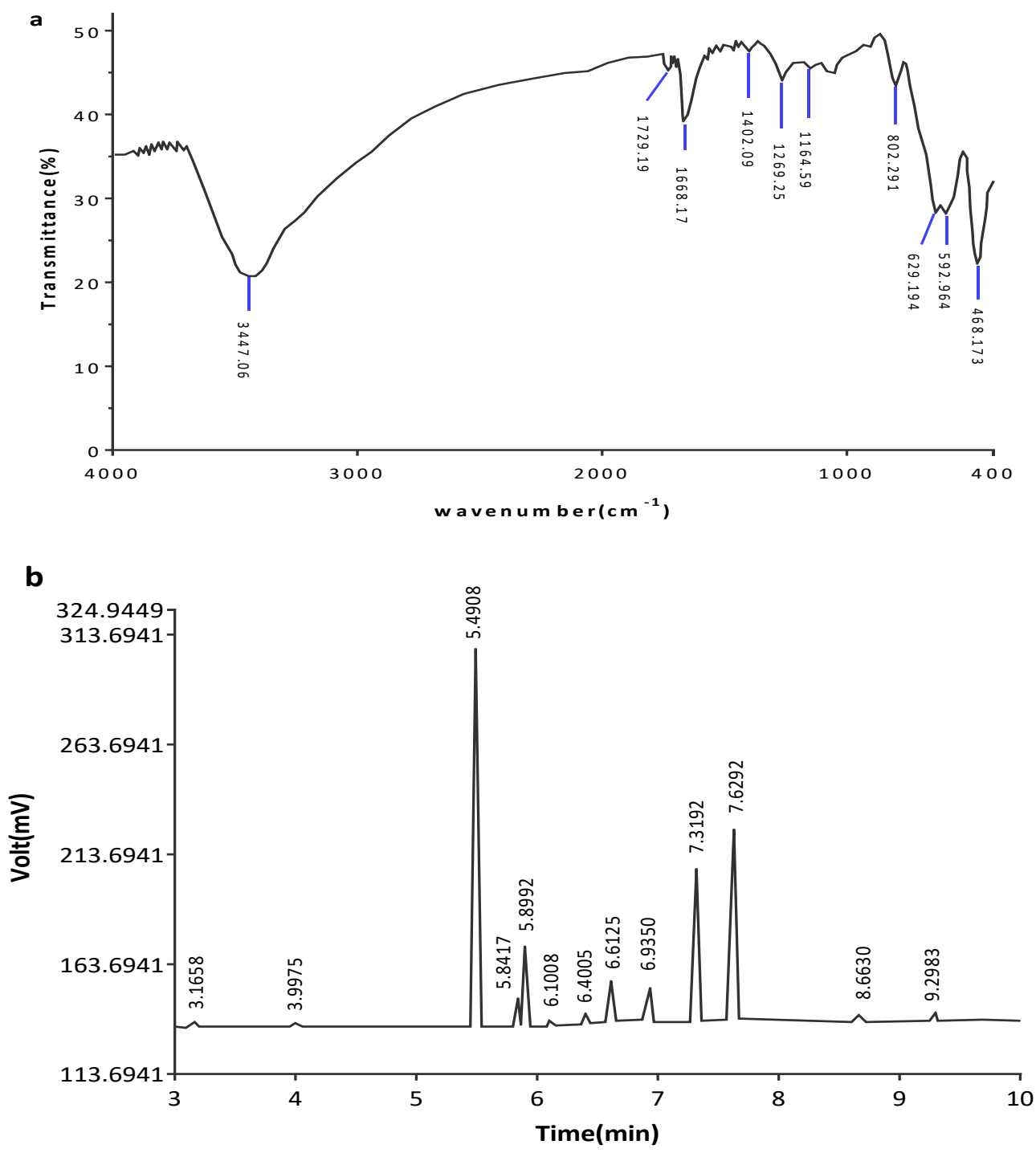

Figure 4. (a) Initial infrared spectrum of microalgae powder; (b) Initial gas chromatogram of lipid extracted from microalgae. 
Table 3. The results of the initial chromatogram of microalgae powder.

\begin{tabular}{l|r|r|r|r|r|r} 
Index & RT $[\mathbf{m i n}]$ & Area[mV*s] & Type & Width[sec] & Area\% & Peak \\
\hline 20 & 7.1825 & 1.6790 & BV & 4.0500 & 0.2918 & C18:2t \\
\hline 21 & 7.2375 & 0.7851 & BB & 5.0500 & 0.1365 & C18:2t \\
\hline 22 & 7.3192 & 76.2294 & BV & 3.5500 & 13.2484 & C18:2c \\
\hline 23 & 7.3683 & 0.3030 & BV & 2.2500 & 0.0527 & \\
\hline 24 & 7.4225 & 0.4895 & BV & 3.1500 & 0.0851 & \\
\hline 25 & 7.4742 & 0.1252 & BV & 2.5000 & 0.0218 & \\
\hline 26 & 7.5225 & 0.1850 & BV & 3.0500 & 0.0322 & \\
\hline 27 & 7.5658 & 0.1612 & BV & 2.6000 & 0.0280 & C20:0 \\
\hline 28 & 7.6292 & 99.9251 & BB & 5.7500 & 17.3666 & C18:3n6
\end{tabular}

The urea crystallization process was used to concentrate GLA; crystallization of urea molecule depends upon fatty acid chain length; the tendency of fatty acids and esters to combine with urea decreases with increasing unsaturation and decreasing chain length. Therefore, the main application of the urea crystallization complex method is to separate the straight-chain compounds from branched ones [32].

\subsection{Final results.}

After the urea crystallization method, IR spectra and gas chromatography was performed again to evaluate the GLA content. Table 4 shows that GLA enrichment was increased in total lipids by $61.17 \%$. GLA enrichment with this method is very suitable, and it should be noted that the amounts of urea and solvents strongly affect it.
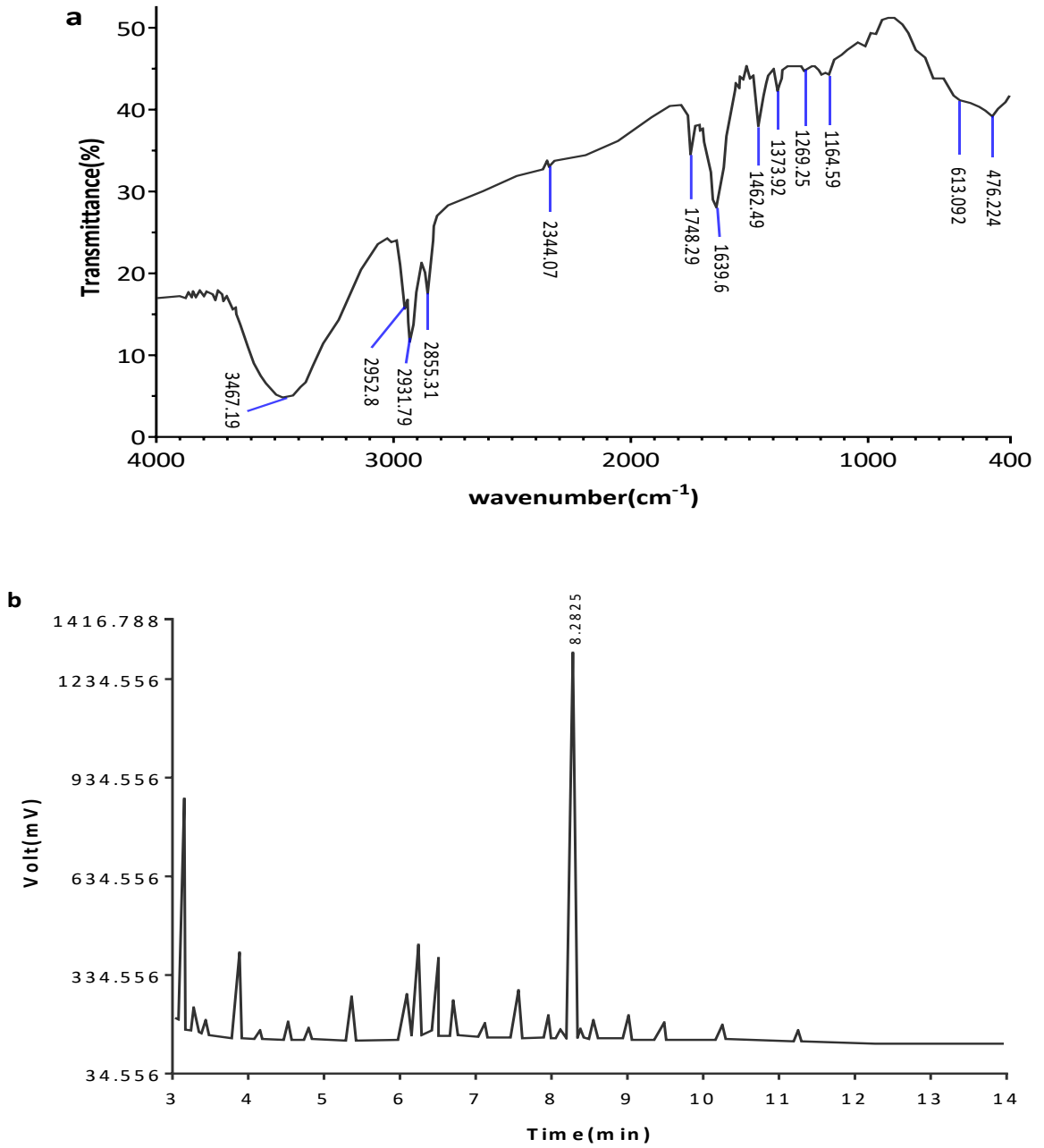

Figure 5. (a) Final infrared spectrum after enrichment; (b) Final gas chromatogram after enrichment. 
Table 4. The result of the final chromatogram after enrichment.

\begin{tabular}{l|l|l|l|l|l|l} 
Index & RT[min] & Area[mV*s] & Type & Width[sec] & Area\% & Peak \\
\hline 12 & 8.1158 & 47.1154 & VV & 8.1500 & 1.1458 & \\
\hline 13 & 8.2050 & 2.3022 & VV & 2.5000 & 0.0560 & \\
\hline 14 & 8.2825 & 2515.5312 & VV & 6.7000 & 61.173 & C18:3n6 \\
\hline 15 & 8.3800 & 49.3673 & VV & 6.1500 & 1.2005 & C20:0 \\
\hline 16 & 8.4858 & 18.0041 & VB & 5.5000 & 0.4378 & C18:3n3 \\
\hline 17 & 8.8142 & 5.6179 & BV & 4.3500 & 0.1366 & C20:1
\end{tabular}

\subsection{Discussion.}

The first action in the case of specific species isolation is to provide a suitable culture medium with optimized environmental conditions [33, 34]. The optimal production of a substance by microalgae requires optimization of their cultivation, which is very important for their commercialization [7]. Therefore, the first phase of this study focused on this issue. GLA and other essential fatty acids have always been considered because of their importance for human health. Biological and pharmaceutical scientists have constantly worked to find new sources and protocols for extracting GLA [1]. Since 1990, studies have begun on the isolation of GLA from microalgae; in that year, Japanese scientists found that $10 \%$ of the fatty acids of Chlorella vulgaris were GLA, and dark conditions could improve its production [35]. Investigating the fatty acid profiles of Chlorella and Spirulina by researchers at Izmir University proved that spirulina potential for GLA extraction is much stronger than Chlorella [36]. Over the past years, research in this regard has focused on optimizing spirulina culture conditions and employing efficient methods for GLA extraction and purification. In 2003, scientists examined different temperatures and nitrogen concentrations, proving that different microalgae conditions induced a different production and subsequently influenced the number of fatty acids, especially GLA. Under optimized conditions, this group obtained a product of $5.2 \mathrm{mg}$ per gram of powder, which is consistent with our rate [37]. Studies continued until Indian scientists enriched the GLA extracted from the ARM 740 strain in 2007. In this study, the Fractionation of lipids was performed using silica gel chromatography. They proved that $94 \%$ of the total GLA was present in microalgae glycolipids and reported recovery of $66 \%$ with a purity of $96 \%$. According to the present study, gas chromatography was used to determine fatty acid composition [38]. A year later, Indian researchers also did another study, which proved that temperature plays an important role in GLA extraction. They were able to isolate $21 \%$ linoleic acid and 18\% GLA at $60^{\circ} \mathrm{C}$ temperature from biomass powder, which is consistent with the results of the initial tests performed in the present study [39]. Spanish scientists studied the isolation of several anti-oxidants and fatty acids from microalgae using supercritical technology and changes in $\mathrm{CO} 2$ and temperature. They even suggested several suitable strains [40]. In 2014, Indian scientists published a review article and provided extraction methods that had been used up to that time [20]. In the present study, except for some of the modified methods, the rest were extracted from this article. The Folch method was performed by L. M. Colla et al. [37] to extract lipid from Spirulina platensis. Rapid and easy processing of large-scale extraction is the major advantage of this method, but it is less sensitive compared with other procedures. Sajilata et al. [22] used another procedure to extract GLA from Spirulina called the Bligh-Dyer method. This method is very efficient, suitable, and costeffective for large-scale extraction. In the present study, modifications were made to improve this method, including adding $1 \% \mathrm{NaCl}$ instead of water to prevent the binding of acidic lipids to denatured lipids. Chloroform is used as the extracting solvent in this method, which is toxic; 
it can cause health risks in large-scale extraction, so it should be ensured that chloroform is completely removed. Guil-Guerrero et al. [21] and Sajilata et al. [22] employed argentite silica gel column chromatography for GLA purification. This method has been shown to be suitable for the isolation of GLA from Spirulina Platensis. This method can obtain Pure GLA, but purifying large quantities of GLA will be expensive and impractical. Other methods, such as low-temperature crystallization [23] and flash chromatography [41], also showcased the good performance of GLA purification, but these methods are expensive and very tedious. In the present study, a very simple and efficient method was performed to purify GLA, called urea crystallization. It requires no organic solvent except ethanol. Therefore, purifying large quantities of GLA is economically feasible. The only problem with this method is the low urea toxicity that should be resolved during the commercialization process. According to the above discussion, studies by researchers worldwide have shown that this issue has received much attention. Due to these products' nutritional and medical uses, novel and optimized methods are constantly being developed for extraction and purification.

\section{Conclusions}

From the results, it can be concluded that the inexpensive and simple methods employed in this paper have high efficiency for the recovery of highly purified GLA, so that it was recovered at a purity of $61.17 \%$. This amount can even increase with further stages of the crystallization of urea. Also, purity could further be increased by altering the ratio of urea, ethanol, and fatty acid esters [42]. This study also highlights the importance of native strains for pharmaceutical and therapeutic applications and malnutrition prevention.

\section{Funding}

This research received no external funding.

\section{Acknowledgments}

The authors gratefully acknowledge the Nano-biotechnology Research Center, Baqiyatallah University of Medical Sciences, Tehran, Iran, and techno-azma laboratory, Tehran, Iran, for supporting us in performing the project.

\section{Conflicts of Interest}

The authors declare no conflict of interest.

\section{References}

1. Matufi F, M.H., Choopani A. Spirulina and Its Role in Immune System: A Review. Journal of Immunology Research and Therapy 2020, 5, 204-211.

2. Choopani, A.; Poursoltan, M.; Fazilati, M.; Latifi, A.M.; Salavati, H. Spirulina: A Source of Gamma-linoleic Acid and its Applications. Journal of Applied Biotechnology Reports 2017, 3, 483-488.

3. Fan, Y.-Y.; Chapkin, R.S. Importance of Dietary $\gamma$-Linolenic Acid in Human Health and Nutrition. The Journal of Nutrition 1998, 128, 1411-1414, https://doi.org/10.1093/jn/128.9.1411.

4. Mendes, R.L.; Reis, A.D.; Pereira, A.P.; Cardoso, M.T.; Palavra, A.F.; Coelho, J.P. Supercritical CO2 extraction of $\gamma$-linolenic acid (GLA) from the cyanobacterium Arthrospira (Spirulina) maxima: experiments and modeling. Chemical Engineering Journal 2005, 105, 147-151, https://doi.org/10.1016/j.cej.2004.10.006.

5. Cameron, M.; Gagnier, J.J.; Chrubasik, S. Herbal therapy for treating rheumatoid arthritis. Cochrane Database of Systematic Reviews 2011, 2, https://doi.org/10.1002/14651858.CD002948.pub2. 
6. Roughan, P.G. Spirulina: A source of dietary gamma-linolenic acid? Journal of the Science of Food and Agriculture 1989, 47, 85-93, https://doi.org/10.1002/jsfa.2740470110.

7. Soltani, N.; Latifi, A.M.; Alnajar, N.; Dezfulian, M.; Shokarvi, S.; Heydari, M.; Choopani, A.J.J.o.A.B.R. Biochemical and Physiological Characterization of Tree Microalgae spp. as Candidates for Food Supplement. 2016, 3, 377-381.

8. Ronda, S.R.; Lele, S. Culture Conditions stimulating high $\gamma$-Linolenic Acid accumulation by Spirulina platensis. Brazilian Journal of Microbiology 2008, 39, 693-697, https://doi.org/10.1590/S151783822008000400018.

9. Moazami, N.; Ranjbar, R.; Ashori, A.; Tangestani, M.; Nejad, A.S. Biomass and lipid productivities of marine microalgae isolated from the Persian Gulf and the Qeshm Island. Biomass and Bioenergy 2011, 35, 19351939, https://doi.org/10.1016/j.biombioe.2011.01.039.

10. Rodrigues, M.S.; Ferreira, L.S.; Converti, A.; Sato, S.; De Carvalho, J.C.M. Influence of ammonium sulphate feeding time on fed-batch Arthrospira (Spirulina) platensis cultivation and biomass composition with and without $\mathrm{pH}$ control. Bioresource technology 2011, 102, 6587-6592, https://doi.org/10.1016/j.biortech.2011.03.088.

11. Gutiérrez-Salmeán, G.; Fabila-Castillo, L.; Chamorro-Cevallos, G. Aspectos nutricionales y toxicológicos de Spirulina (arthrospira). Nutricion hospitalaria 2015, 32, 34-40, https://doi.org/10.3305/nh.2015.32.1.9001.

12. Mühling, M.; Belay, A.; Whitton, B.A. Variation in fatty acid composition of Arthrospira (Spirulina) strains. Journal of Applied Phycology 2005, 17, 137-146, https://doi.org/10.1007/s10811-005-7213-9.

13. Golmakani, M.-T.; Rezaei, K.; Mazidi, S.; Razavi, S.H. $\gamma$-Linolenic acid production by Arthrospira platensis using different carbon sources. European Journal of Lipid Science and Technology 2012, 114, 306-314, https://doi.org/10.1002/ejlt.201100264.

14. Ronda, S.R.; Bokka, C.S.; Ketineni, C.; Rijal, B.; Allu, P.R. Aeration effect on Spirulina platensis growth and $\gamma$-linolenic acid production. Brazilian Journal of Microbiology 2012, 43, 12-20, https://doi.org/10.1590/S1517-83822012000100002.

15. Colla, L.M.; Oliveira Reinehr, C.; Reichert, C.; Costa, J.A.V. Production of biomass and nutraceutical compounds by spirulina platensis under different temperature and nitrogen regimes. Bioresource Technology 2007, 98, 1489-1493, https://doi.org/10.1016/j.biortech.2005.09.030.

16. Casazza, A.A.; Ferrari, P.F.; Aliakbarian, B.; Converti, A.; Perego, P. Effect of UV radiation or titanium dioxide on polyphenol and lipid contents of Arthrospira (Spirulina) platensis. Algal Research 2015, 12, 308315, https://doi.org/10.1016/j.algal.2015.09.012.

17. Prabakaran, P.; Ravindran, A.D. A comparative study on effective cell disruption methods for lipid extraction from microalgae. Letters in applied microbiology 2011, 53, 150-154, https://doi.org/10.1111/j.1472765X.2011.03082.X.

18. Lee, J.-Y.; Yoo, C.; Jun, S.-Y.; Ahn, C.-Y.; Oh, H.-M. Comparison of several methods for effective lipid extraction from microalgae. Bioresource technology 2010, 101, S75-S77, https://doi.org/10.1016/j.biortech.2009.03.058.

19. Mendes, R.L.; Reis, A.D.; Palavra, A.F. Supercritical CO2 extraction of $\gamma$-linolenic acid and other lipids from Arthrospira (Spirulina) maxima: Comparison with organic solvent extraction. Food Chemistry 2006, 99, 5763, https://doi.org/10.1016/j.cej.2004.10.006.

20. Ranjith Kumar, R.; Hanumantha Rao, P.; Arumugam, M. Lipid extraction methods from microalgae: a comprehensive review. Frontiers in Energy Research 2015, 2, 61, https://doi.org/10.3389/fenrg.2014.00061.

21. Guil-Guerrero, J.L.; Campra-Madrid, P.; Belarbi, E.-H. $\gamma$-Linolenic acid purification from seed oil sources by argentated silica gel chromatography column. Process Biochemistry 2000, 36, 341-354, https://doi.org/10.1016/s0032-9592(00)00217-X.

22. Sajilata, M.; Singhal, R.; Kamat, M. Fractionation of lipids and purification of $\gamma$-linolenic acid (GLA) from Spirulina platensis. Food Chemistry 2008, 109, 580-586, https://doi.org/10.1016/j.foodchem.2008.01.005.

23. López-Martínez, J.C.; Campra-Madrid, P.; Guil-Guerrero, J.L. $\gamma$-Linolenic acid enrichment from Borago officinalis and Echium fastuosum seed oils and fatty acids by low temperature crystallization. Journal of bioscience and bioengineering 2004, 97, 294-298, https://doi.org/10.1016/S1389-1723(04)70208-X.

24. Nagao, T.; Watanabe, Y.; Kobayashi, T.; Sumida, M.; Kishimoto, N.; Fujita, T.; Shimada, Y. Enzymatic purification of dihomo- $\gamma$-linolenic acid from Mortierella single-cell oil. Journal of Molecular Catalysis B: Enzymatic 2007, 44, 14-19, https://doi.org/10.1016/j.molcatb.2006.08.006.

25. Bligh, E.G.; Dyer, W.J. A Rapid Method of Total Lipid Extraction and Purification. Canadian Journal of Biochemistry and Physiology 1959, 37, 911-917, https://doi.org/10.1139/o59-099. 
26. Takagi, M.; Yoshida, T. Effect of salt concentration on intracellular accumulation of lipids and triacylglyceride in marine microalgae Dunaliella cells. Journal of bioscience and bioengineering 2006, 101, 223-226, https://doi.org/10.1263/jbb.101.223.

27. Seifi Kafshgari, H.; Yazdanian, M.; Ranjbar, R.; Tahmasebi, E.; Mirsaeed, S.; Tebyanian, H.; Ebrahimzadeh, M.A.; Goli, H.R. The effect of Citrullus colocynthis extracts on Streptococcus mutans, Candida albicans, normal gingival fibroblast and breast cancer cells. J Biol Res 2019, 92, 8201, https://doi.org/10.4081/jbr.2019.8201.

28. Kariminik, A.; Moradalizadeh, M.; Foroughi, M.M.; Tebyanian, H.; Motaghi, M.M. Chemical Composition and Antibacterial Activity of the Essential Oils Extracted From 4 Medicinal Plants (Labiatae) of Kerman, Iran. Appl. Biotechnol. Rep 2019, 6, 172-179, https://doi.org/10.29252/JABR.06.04.07.

29. Tavakolizadeh, M.; Pourjavadi, A.; Ansari, M.; Tebyanian, H.; Seyyed Tabaei, S.J.; Atarod, M.; Rabiee, N.; Bagherzadeh, M.; Varma, R.S. An environmentally friendly wound dressing based on a self-healing, extensible and compressible antibacterial hydrogel. Green Chemistry 2021, https://doi.org/10.1039/d0gc02719g.

30. Yazdanian, M.; Tabesh, H.; Houshmand, B.; Tebyanian, H.; Soufdoost, R.S.; Tahmasebi, E.; Karami, A.; Ghullame, S. Fabrication and properties of $\beta \mathrm{TCP} /$ Zeolite/Gelatin scaffold as developed scaffold in bone regeneration: in vitro and in vivo studies. Biocybern Biomed Eng 2020, 40, 1626-1637, https://doi.org/https://doi.org/10.1016/j.bbe.2020.10.006.

31. Mosaddad, S.A.; Yazdanian, M.; Tebyanian, H.; Tahmasebi, E.; Yazdanian, A.; Seifalian, A.; Tavakolizadeh, M. Fabrication and properties of developed collagen/strontium-doped Bioglass scaffolds for bone tissue engineering. J. Mater. Res. Technol 2020, 9, 14799-14817, https://doi.org/10.1016/j.jmrt.2020.10.065.

32. Ubaid Ahmed, S.; Konda Reddy, K.; Swathy, S.L.; Singh, S.K.; Kanjilal, S.; Prasad, R.B.N.; Pandey, A. Enrichment of $\gamma$-linolenic acid in the lipid extracted from Mucor zychae MTCC 5420. Int. Food Res. J 2009, 42, 449-453, https://doi.org/10.1016/j.foodres.2009.01.002.

33. Mosaddad, S.A.; Beigi, K.; Doroodizadeh, T.; Haghnegahdar, M.; Golfeshan, F.; Ranjbar, R.; Tebyanian, H. Therapeutic applications of herbal/synthetic/bio-drug in oral cancer: An update. Eur. J. Pharmacol 2021, 890, 173657, https://doi.org/https://doi.org/10.1016/j.ejphar.2020.173657.

34. Moghadam, E.T.; Yazdanian, M.; Tahmasebi, E.; Tebyanian, H.; Ranjbar, R.; Yazdanian, A.; Seifalian, A.; Tafazoli, A. Current herbal medicine as an alternative treatment in dentistry: In vitro, in vivo and clinical studies. Eur. J. Pharmacol 2020, 889, 173665, https://doi.org/https://doi.org/10.1016/j.ejphar.2020.173665.

35. Hirano, M.; Mori, H.; Miura, Y.; Matsunaga, N.; Nakamura, N.; Matsunaga, T. $\gamma$-linolenic acid production by microalgae. Applied Biochemistry and Biotechnology 1990, 24-25, 183-191, https://doi.org/10.1007/bf02920244.

36. Ötleş, S.; Pire, R. Fatty acid composition of Chlorella and Spirulina microalgae species. Journal of AOAC international 2001, 84, 1708-1714.

37. Colla, L.M.; Bertolin, T.E.; Costa, J.A.V. Fatty Acids Profile of Spirulina platensis Grown Under Different Temperatures and Nitrogen Concentrations. Zeitschrift für Naturforschung C 2004, 59, 55-59, https://doi.org/10.1515/znc-2004-1-212.

38. Mahajan, G.; Kamat, M. $\gamma$-Linolenic acid production from Spirulina platensis. Applied Microbiology and Biotechnology 1995, 43, 466-469, https://doi.org/10.1007/BF00218450.

39. Chaiklahan, R.; Chirasuwan, N.; Loha, V.; Bunnag, B. Lipid and fatty acids extraction from the cyanobacterium spirulina. ScienceAsia 2008, 34, 299, https://doi.org/10.2306/scienceasia15131874.2008.34.299.

40. Cardoso, L.C.; Serrano, C.M.; Rodríguez, M.R.; de la Ossa, E.J.M.; Lubián, L.M. Extraction of Carotenoids and Fatty Acids from Microalgae Using Supercritical Technology. American Journal of Analytical Chemistry 2012, 03, 877-883, https://doi.org/10.4236/ajac.2012.312A116.

41. Jubie, S.; Dhanabal, S.; Chaitanya, M. Isolation of methyl gamma linolenate from spirulina platensis using flash chromatography and its apoptosis inducing effect. BMC complementary and alternative medicine $\mathbf{2 0 1 5}$, 15, 263, https://doi.org/10.1186/s12906-015-0771-8.

42. Mosaddad, S.A.; Salari, Y.; Amookhteh, S.; Soufdoost, R.S.; Seifalian, A.; Bonakdar, S.; Safaeinejad, F.; Moghaddam, M.M.; Tebyanian, H. Response to Mechanical Cues by Interplay of YAP/TAZ Transcription Factors and Key Mechanical Checkpoints of the Cell: A Comprehensive Review. Cell Physiol Bio Chem 2021, 55, 33-60, https://doi.org/10.33594/000000325. 